\title{
Antitumor effect of 1, 8-cineole against colon cancer
}

\author{
SOICHIRO MURATA ${ }^{1,2}$, RISA SHIRAGAMI $^{1}$, CHIHIRO KOSUGI $^{1}$, TOHRU TEZUKA $^{1}$, MASATO YAMAZAKI $^{1}$, \\ ATSUSHI HIRANO ${ }^{1}$, YUKINO YOSHIMURA ${ }^{1}$, MASATO SUZUKI ${ }^{1}$, KIYOHIKO SHUTO ${ }^{1}$, \\ NOBUHIRO OHKOHCHI ${ }^{2}$ and KEIJI KODA ${ }^{1}$
}

\author{
${ }^{1}$ Department of Surgery, Teikyo University Chiba Medical Center, Ichihara; ${ }^{2}$ Department of Surgery, Division of \\ Gastroenterological and Hepatobiliary Surgery, and Organ Transplantation, University of Tsukuba, Tsukuba, Ibaraki, Japan
}

Received June 28, 2013; Accepted August 1, 2013

DOI: 10.3892/or.2013.2763

\begin{abstract}
Several essential oils possess pharmacological effects. Among the various constituents of essential oils, 1,8-cineole has been shown to possess pharmacological effects such as anti-bacterial and anti-inflammatory effects. The effect of 1,8-cineole on human colorectal cancer cells, however, has not reported previously. In this study, we have investigated the anti-proliferative effect of 1,8-cineole on human colon cancer cell lines HCT116 and RKO by WST- 8 and BrdU assays. The cytotoxicity of 1,8-cineole was investigated by $\mathrm{LDH}$ activity and TUNEL staining. The mechanism of apoptosis by 1,8 -cineole was determined by western blot analyses. In in vivo study, RKO cells were injected into the SCID mice and the effect of 1,8-cineole was investigated. Specific induction of apoptosis, not necrosis, was observed in human colon cancer cell lines HCT116 and RKO by 1, 8-cineole. The treatment with 1, 8-cineole was associated with inactivation of survivin and Akt and activation of $\mathrm{p} 38$. These molecules induced cleaved PARP and caspase-3, finally causing apoptosis. In xenotransplanted SCID mice, the 1,8-cineole group showed significantly inhibited tumor progression compared to the control group. These results indicated 1,8-cineole suppressed human colorectal cancer proliferation by inducing apoptosis. Based on these studies 1,8-cineole would be an effective strategy to treat colorectal cancer.
\end{abstract}

\section{Introduction}

The use of plant-derived natural products for medical benefits is playing an important role globally. Several anticancer drugs available on the market today such as Taxol, Oncovin, Navelbine and Vumon trace their origins to plants (1-3). In cancer therapy, the focus is on strategies that suppress tumor

Correspondence to: Dr Soichiro Murata, Department of Surgery, Division of Gastroenterological and Hepatobiliary Surgery, and Organ Transplantation, University of Tsukuba, Tsukuba, Ibaraki, Japan E-mail: soichiro@md.tsukuba.ac.jp

Key words: colon cancer 1, 8-cineole apoptosis growth through cell cycle disruption (4) and activate the apoptotic program in the cell (5).

Several essential oils from plants possess medical benefits. Among the various constituents of essential oils, 1, 8-cineole has been shown to possess pharmacological effects. The content of 1,8-cineole in the essential oils varies in the different Eucalyptus species, from 25 to $90 \%(6,7)$. 1, 8-cineole has been used as a percutaneous penetration enhancer (8), as an antibacterial and expectorant $(6)$, anti-inflammatory $(9,10)$ or antihypertensive (11) agent. 1,8-cineole was reported to induce apoptosis in leukemia cell lines (12).

In this study, we found that 1,8-cineole exerts antitumor activity on human colon cancer cell lines HCT116 and RKO. We investigated whether 1,8-cineole induces apoptosis in two human colorectal cancer cell lines and in a xenograft model.

\section{Materials and methods}

Reagents. 1, 8-cineole (eucalyptol) was obtained from Sigma (St. Louis, MO, USA). For cell culture experiment, 1, 8-cineole was dissolved in ethanol and then in water at a concentration of $0.1 \mathrm{mg} / \mathrm{ml}$. In an in vitro experiment, 5-50 mM of 1, 8-cineole was added to the medium and cell viability assay and western blot experiments were performed $24 \mathrm{~h}$ later.

Cells. The human CRC cell lines HCT116 and RKO were used and tested for mycoplasma-free cell lines. The cancer cell lines were subdivided into multiple tubes for stock in liquid nitrogen immediately after possession. All cell lines were subjected to the present experiment within 6 months of resuscitation. Stock cultures were grown in high-glucose DMEM containing $10 \%$ FBS and $1 \%$ antibiotics. The cells were grown in growth medium at $37^{\circ} \mathrm{C}$ in a $95 \%$ air, $5 \% \mathrm{CO}_{2}$-humidified incubator.

Cell viability assay. To measure the cytotoxicity of 1,8-cineole against these cancer cells, $3 \times 10^{3}$ cells were plated per well onto 96-well plates. Following overnight culture, 1, 8-cineole and oxaliplatin were added at specified concentrations. After $24 \mathrm{~h}$ incubation, cell viability was measured by the mitochondrial activity in reducing 2-(2-methoxy-4-nitrophenyl)-3-(4-nitrophenyl)-5-(2,4-disulfophenyl)-2H-tetrazolium monosodium salt (WST-8) to formazan using a Cell Counting kit-8 (Dojindo Laboratories, Kumamoto, Japan). Cells were incubated with a 
reagent as per the manufacturer's instructions. Plates were read at $\mathrm{A} 450$ on a spectrometer.

Cell proliferation assay. To measure the cell proliferation activity of 1,8-cineole against HCT116 and RKO cancer cells, $3 \times 10^{3}$ cells were plated per well onto 96 -well plates. Following overnight culture, 1, 8-cineole was added at specified concentrations. After $24 \mathrm{~h}$ of incubation, cell proliferation was measured with a BrdU assay kit (Roche Diagnostics, Penzberg, Germany). Cells were incubated with a reagent as per the manufacturer's instructions. Plates were read at A450 on a spectrometer.

Lactate dehydrogenase ( $L D H)$ activity assay. In order to evaluate the activity of the cytoplasmic enzyme lactate dehydrogenase ( $\mathrm{LDH}$ ) released from the cytosol when cells were damaged under stress, HCT116 and RKO cells were seeded $\left(1 \times 10^{5}\right.$ cells $\left./ \mathrm{ml}\right)$ on 96 -well plates. The LDH activity was determined using a commercial kit (Takara Bio, Tokyo, Japan). Absorbance values were then correlated with the number of viable cells to predict the cytotoxic activity. Triton X-100 (1\%) (Sigma) was used as a positive control.

Apoptosis assay. The In Situ Cell Death Detection kit (Roche Diagnostics, Basel, Switzerland) was used for the demonstration of apoptotic cell death of cell culture and liver tissue. Cells $\left(3 \times 10^{4}\right)$ were plated per well onto Lab-Tek II Chamber Slides (Nalge Nunc International, Tokyo, Japan) and were incubated with the terminal deoxynucleotidyl transferase-mediated dUTP nick end labeling (TUNEL) reaction mixture according to the manufacturer's recommendations.

Western blot analysis. For western blot analysis, total protein extracts of HCT116 cells were obtained $24 \mathrm{~h}$ after 1, 8-cineole treatment and separated by $10 \%$ SDS-PAGE and transferred to nitrocellulose membrane (Millipore, Bedford, MA, USA). The following antibodies were used as primary antibodies: cleaved caspase-3 (9661), cleaved poly(ADP-ribose) polymerase (PARP) (5625p), survivin (2808p), phosphoserine 473 Akt (9271), phospho p38 (4511p) and GAPDH (2118) (Cell Signaling Technology, Beverly, MA, USA). Secondary goat anti-rabbit antibody conjugated with horseradish peroxidase was purchased from Cell Signaling Technology.

Animals. Seven-week-old male severe combined immunodeficiency (SCID) mice (Clea, Tokyo, Japan), weighing 24-28 g, were utilized. The mice were kept in a temperature-controlled room on a 12-h light-dark cycle. They had free access to water and standard chow throughout the experiment. After an acclimation period of $\geq 7$ days, the mice were separated into two groups as follows: control group, mice without any treatment $(n=10)$; and 1, 8-cineole group, mice with 1, 8-cineole treatment $(n=9)$. All animal experiments were carried out in a humane manner after receiving approval from the Institutional Animal Committee of Teikyo University and in accordance with the Regulation for Animal Experiments of the University and Fundamental Guidelines for Proper Conduct of Animal Experiments and Related Activities in Academic Research Institutions under the jurisdication of the Ministry of Education, Culture, Sports, Science and Technology of Japan.
Xenograft. Cells $\left(2 \times 10^{6}\right)$ of RKO were injected subcutaneously into the right flank of each mouse with a 27 -gauge needle. Tumors were detected by palpation and measured periodically with calipers. Seven days after tumor injection, $50 \mathrm{mg} /$ $\mathrm{kg}$ of 1,8-cineole was injected subcutaneously every 3 days. Twenty-one days after inoculation, mice were sacrificed and tumors were removed for examination.

Statistical analysis. All data are expressed as the mean \pm SD of samples. Comparisons between various points were made using one-way ANOVA. Comparisons between the two groups were made using Mann-Whitney U test. Significant data were examined by the Bonferroni-Dunn multiple comparisons post hoc test. In all cases, a P-value $<0.05$ was considered statistically significant.

\section{Results}

Dose-dependent inhibition of proliferation of human CRC cell lines by 1,8-cineole. We initially determined whether 1,8-cineole treatment led to the inhibition of human CRC cell proliferation. $\mathrm{CRC}$ cells were treated with various doses of 1,8-cineole for $24 \mathrm{~h}$ and cell viability was assayed using WST-8 assay (Fig. 1A and B) and BrdU assay (Fig. 1C and D). Fig. 1 shows that as the dose of 1,8-cineole increased from 5 to $50 \mathrm{mM}$, cell growth inhibition increased in a dosedependent fashion in CRC cell lines HCT116 and RKO. 1,8-cineole-induced growth inhibition was found to be statistically significant $(\mathrm{p}<0.01)$ (one-way ANOVA) in $5-50 \mathrm{mM}$ of 1, 8-cineole compared to $0 \mathrm{mM}$ in WST-8 assay and 5-25 $\mathrm{mM}$ in BrdU assay.

1, 8-cineole does not induce necrosis. Fig. 2 shows the cytoplasmic LDH released of HCT116 and RKO cells treated with each sample at 5-50 mM of 1,8-cineole. Compared to culture medium (negative control), no statically significant increase in LDH released by these cells was observed. However, all samples showed a statistically significant difference $(\mathrm{p}<0.01)$ in $\mathrm{LDH}$ released when compared to $1 \%$ Triton X-100 (positive control). Damaged cells or those under stress can release cytoplasmic LDH and other substances into the medium due to disruption of the cytoplasmic membrane and cell necrosis (13). Therefore, it is possible that the cytotoxic activity of the samples was not based on the mechanism of cell death by necrosis. Consequently, these results indicate that the cytotoxic activity of the samples can involve pathways inducing cell death by apoptosis.

Induction of apoptosis via activation of caspase-dependent pathway by 1, 8-cineole. In subsequent experiments, we determined the mechanism of the observed suppressive effect of 1, 8-cineole by WST- 8 and BrdU assays. The overexpression of survivin has been observed to cooperate with survival pathways, including the phosphatidylinositol-3-kinase (PI3K)/Akt pathway. Suppression of survivin and constitutive activated Akt and activation of p38 was observed in 12.5-50 mM of 1, 8-cineole. These molecules induced increments of cleaved PARP and caspase-3 in 1, 8-cineole treatment (Fig. 3A). TUNEL staining of RKO cells showed apoptotic cells in $50 \mathrm{mM}$ of 1, 8-cineole (Fig. 3B). 

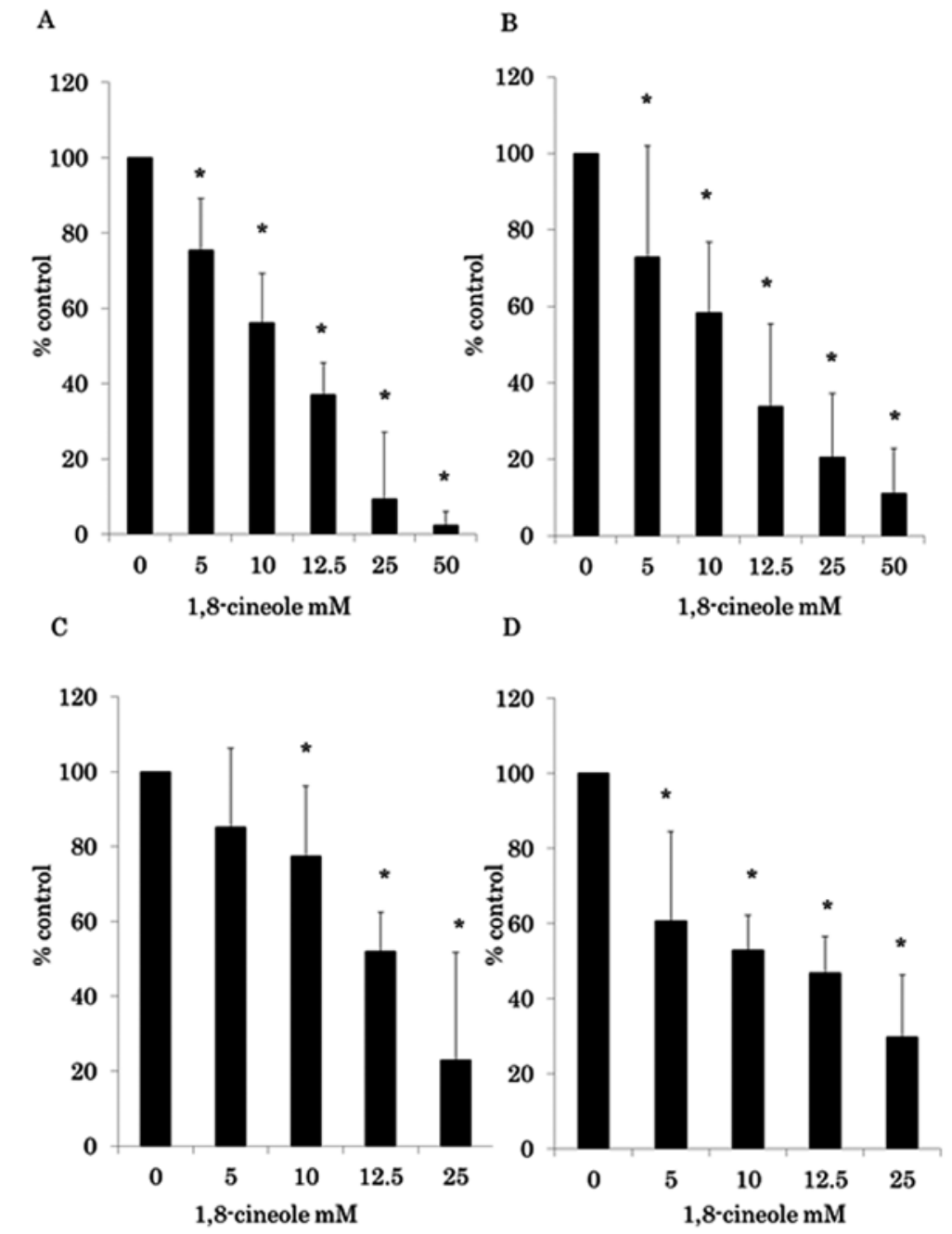

Figure 1. Effect of 1,8-cineole on the WST-8 assay and BrdU assay of human CRC cell lines. CRC cell lines were treated with 0-50 mM 1, 8-cineole for $24 \mathrm{~h}$. (A) RKO; (B) HCT116. "p<0.01 compared to $0 \mathrm{mM} \mathrm{1,8-cineole.} \mathrm{The} \mathrm{values} \mathrm{indicate} \mathrm{ratio} \mathrm{compared} \mathrm{to} 0 \mathrm{mM} 1,8$-cineole as $100 \%$ control. In the BrdU assay of human CRC cell lines, cell lines were treated with 0-25 mM 1, 8-cineole for $24 \mathrm{~h}$. (C) RKO; (D) HCT116. "p<0.01 compared to $0 \mathrm{mM} \mathrm{1,8-cineole.} \mathrm{The} \mathrm{values}$ indicate ratio compared to $0 \mathrm{mM} 1,8$-cineole as $100 \%$ control.
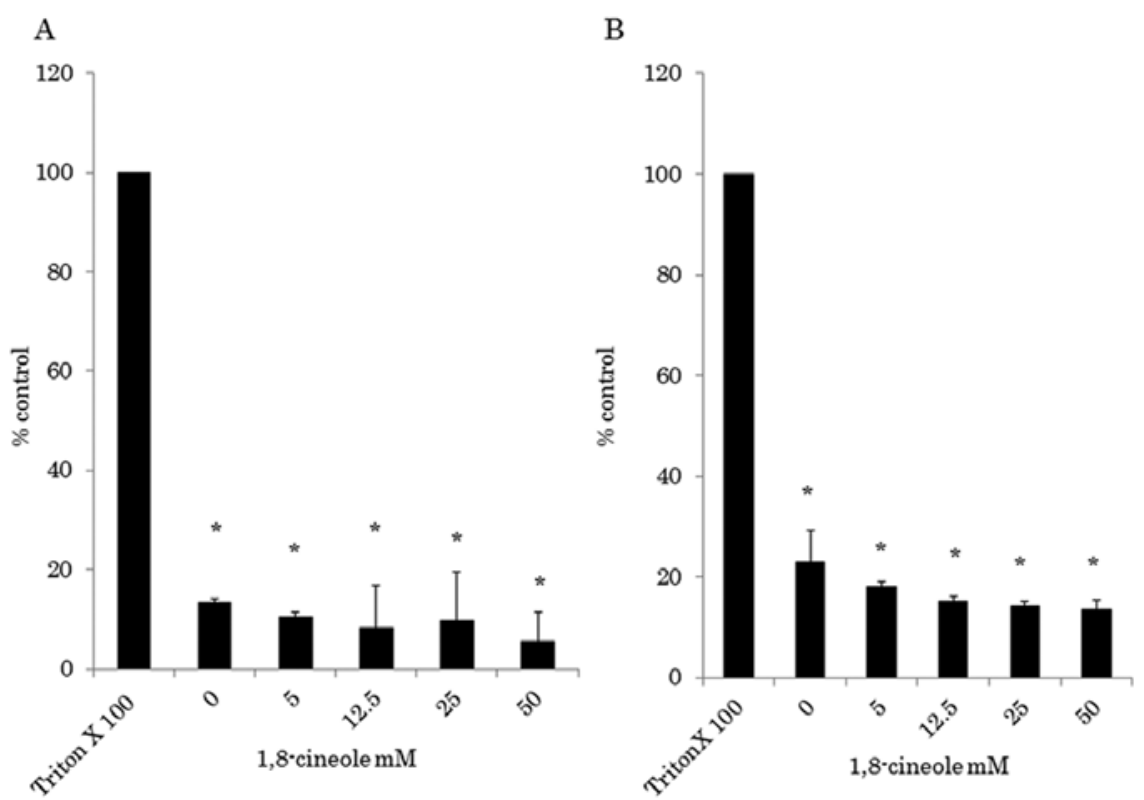

Figure 2. Effect of 1, 8-cineole on the LDH activity assay of human CRC cell lines. CRC cell lines were treated with 0-50 mM 1, 8-cineole for $24 \mathrm{~h}$. (A) RKO; (B) HCT116. ${ }^{*} \mathrm{p}<0.01$ compared to $1 \%$ Triton $\mathrm{X}-100$. The values indicate ratio compared to $1 \%$ Triton $\mathrm{X}-100$ as $100 \%$ control. 
A

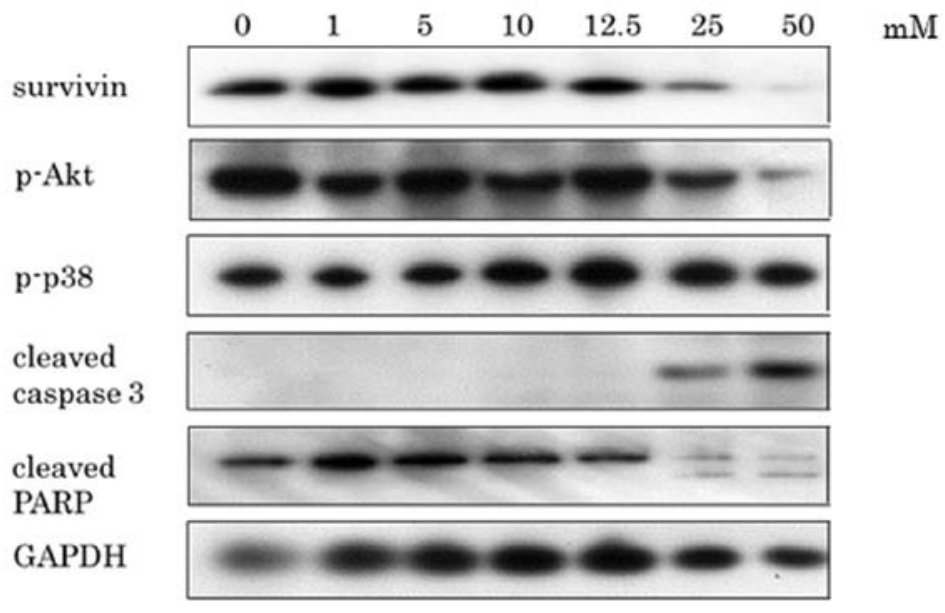

B

0

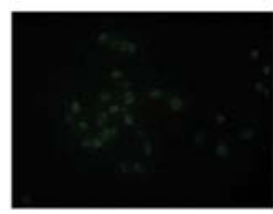

5

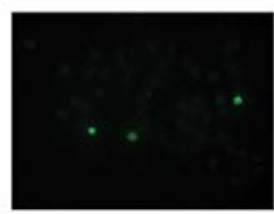

50

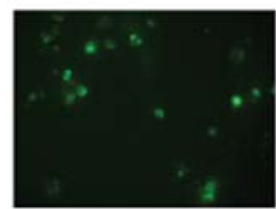

Figure 3. (A) RKO cells were treated with 0-50 mM of 1, 8-cineole for $24 \mathrm{~h}$. After cell lysis, equal amounts of proteins were separated by SDS-PAGE, transferred to Immobilon membrane and immunoblotted with antibodies against survivin, p-Akt, p-p38, cleaved caspase-3, cleaved PARP and GAPDH as indicated. 1, 8-cineole treatment caused suppression of survivin, dephosphorylation of constitutive phosphorylation of Akt, p38 activation and cleavage of PARP and caspase-3. (B) TUNEL staining of RKO cells, which were treated with 0,5 and $50 \mathrm{mM}$ of 1,8-cineole. Stained cells indicate TUNEL positive apoptotic cells.

A

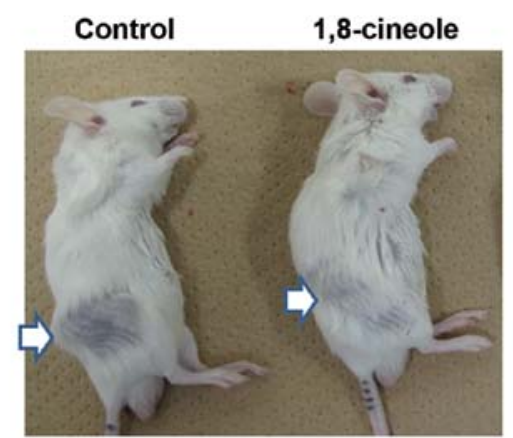

C

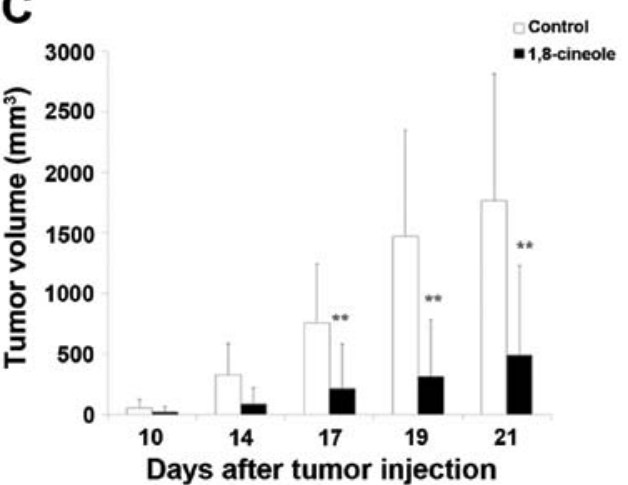

B

Control

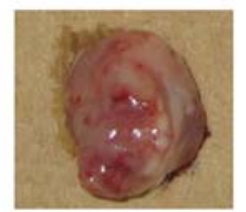

D

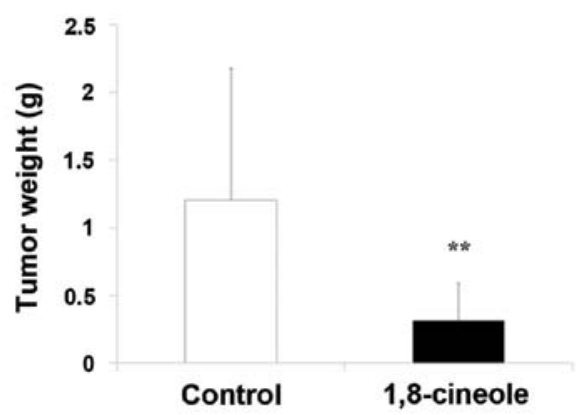

Figure 4. 1, 8-cineole therapy significantly inhibits tumor growth of the xenograft RKO tumors in SCID mice. RKO cells were injected into the right flank subcutaneously. Seven days later, $50 \mathrm{mg} / \mathrm{kg}$ of 1,8-cineole was injected subcutaneously 4 times, with 3-day intervals. Control animals were prepared as no treatment. (A) Representative mice of the 2 groups. Arrows indicated tumors. No skin damage was observed in the 2 groups. (B) Representative xenograft tumors of the 2 groups. (C) Tumor volume of the 2 groups. Columns, mean; bars, SD. White bar, control group. Black bar, 1, 8-cineole group. "p $<0.05$ versus control group. (D) Tumor weight of the 2 groups. ${ }^{* *} \mathrm{p}<0.01$ versus control group. 
1, 8-cineole inhibits tumor growth of RKO xenografts. We evaluated the potential effectiveness of 1,8-cineole in a xenograft model of RKO, which was subcutaneously injected into the right flank of each mouse. Fig. 4A shows representative mice of each group. No skin damage was observed in the 1,8-cineole group. Fig. 4B shows tumors removed from the representative control and mice with 1,8-cineole groups. Fig. 4C indicates tumor growth curve. Fig. 4D indicates tumor weight in the 2 groups. Tumor growth was significantly inhibited in the 1,8-cineole group, compared to the control group.

\section{Discussion}

Essential oils and their components have been investigated regarding their effect against a variety of human cancer cell lines. Lemon balm was verified to show cytotoxic activity against some human cancer cell lines and a mouse cell line (14). The genus Eucalyptus is native to Australia and this plant is mainly cultivated for paper, pharmaceutical and cosmetics industries. Several species of Eucalyptus are also used in traditional medicine as antiseptics and against infections of the upper respiratory tract such as the common cold, influenza and sinus congestion $(15,16)$. Essential oils from this species have a therapeutic application in the treatment of pulmonary infections by inhalation (17) and the monoterpene extracted from Eucalyptus species exhibit antibacterial activity (18). Ashour (19) showed cytotoxic activities of volatile oils and extracts from Eucalyptus species against the human breast adenocarcinoma cell line MCF7.

Among the various constituents of essential oils extracted from Eucalyptus species, 1, 8-cineole is one of the main constituent in the essential oils from Eucalyptus species $(6,7)$. 1, 8-cineole has been shown to possess several pharmacological effects. The cytotoxic activities against tumor cell lines were previously only reported in leukemia cell lines (12). In this study, we revealed the cytotoxic activities of 1,8-cineole against human colorectal cancer for the first time.

The mechanism of cell killing by 1,8-cineole is not fully understood. Suppression of growth by 1,8-cineole in the leukemia cell lines was reported to the induction of apoptosis (12). Survivin is a type of inhibitor of apoptosis (IAP) protein and helps repress apoptosis. IAPs are highly expressed in most malignancies and play key roles in the survival of neoplastic cells by negatively regulating apoptosis (20). Several studies show that high survivin expression in tumors is associated with lower apoptosis index, increased resistance to chemotherapeutic agents and poor patient survival (21). Specific inhibition of survivin expression in multiple cancer cell lines by a survivin antisense oligonucleotide induced caspase-3-dependent apoptosis, cell cycle arrest in the $\mathrm{G}_{2}-\mathrm{M}$ phase and multinucleated cells (21). Inhibition of survivin expression using 1, 8-cineole mediates the apoptosis and growth arrest in human colorectal cancers. p38 MAP kinase is a member of the MAP kinase family and is activated by a variety of cellular stresses (22-26). Activated p38 MAP kinase appears to have multiple targets in the apoptotic pathway. Activated p38 mediates apoptosis by the downregulation of survivin in colon cancer cells $(27,28)$. The phosphatidylinositol-3-kinase (PI3K)/Akt pathway is a cell signaling pathway and signaling through the PI3K/Akt signaling axis promotes growth and survival and undergoes apoptosis (29). The overexpression of PI3K/Akt pathway was observed in many human cancers (30). Our results show that human CRC cell line RKO contains constitutively active PI3K/ Akt pathway. RKO expressed phosphoserine 473-Akt constitutively and treatment with 1,8-cineole dephosphorylated Akt. 1,8-cineole treatment activated p38 and dephosphorylated Akt, which induced caspase- 3 cleavage and resultant cleavage of PARP and finally caused apoptosis.

In a xenotransplant mouse model, 1, 8-cineole therapy showed tumor shrinkage comparable to the control group. Throughout the experiments, the animals had no weight loss in 1,8-cineole and control groups (data not shown). Moreover, we could not observe any skin damage in the 1,8-cineole group, which was injected locally.

In conclusion, these findings demonstrate that 1, 8-cineole might exert its antitumor activity by triggering apoptosis in human colorectal cancer cells in vitro and in vivo. 1, 8-cineole shows promise as a strong and safe chemotherapeutic agent for colorectal cancer.

\section{Acknowledgements}

The authors thank Satoko Nakabayashi for technical assistance. This study was supported in part by grants-in-aid from the Ministry of Education, Culture, Sports, Science and Technology of Japan (MEXT).

\section{References}

1. Pezzuto JM: Plant-derived anticancer agents. Biochem Pharmacol 53: 121-133, 1997.

2. Kinghorn AD, Farnsworth NR, Doel Soejarto D, et al: Novel strategies for the discovery of plant-derived anticancer agents. Pure Appl Chem 71: 1611-1618, 1999.

3. Lee KH: Anticancer drug design based on plant-derived natural products. J Biomed Sci 6: 236-250, 1999.

4. Moalic S, Liagre B, Corbiére C, et al: A plant steroid, diosgenin, induces apoptosis, cell cycle arrest and COX activity in osteosarcoma cells. FEBS Lett 506: 225-230, 2001.

5. Fan S, Cherney B, Reinhold W, et al: Disruption of p53 function in immortalized human cells does not affect survival or apoptosis after taxol or vincristine treatment. Clin Can Res 4: 1047-1054, 1998.

6. Giamakis A, Kretsi O, Chinou IB, et al: Eucalyptus camaldulensis: volatiles from immature flowers and high production of 1,8-cineole and b-pinene by in vitro cultures. Phytochemistry 58 : 351-355, 2001.

7. Tsiri DO, Kretsi O, Chinou IB, et al: Composition of fruit volatiles and annual changes in the volatiles of leaves of Eucalyptus camandulensis Dehn. Growing in Greece. Flav Fragr J 18: 244-247, 2003.

8. Levinson KK, Takayama K, Okabe K, et al: Formulation optimization of indomethacine gels containing a combination of three kinds of cyclic monoterpenes as percutaneous penetration enhancers. J Pharm Sci 83: 1367-1372, 1994.

9. Juergens UR, Stober M, Schmidt-Schilling L, et al: Antiinflammatory effects of encalyptol (1,8-cineole) in bronchial asthma:inhibition of arachidonic acid metabolism in human blood monocytes ex vivo. Eur J Med Res 17: 407-412, 1998.

10. Juergens UR, Stober M and Vetter H: Inhibition of cytokine production and arachidonic acid metabolism by eucalyptol $(1,8-$ cineole) in human blood monocytes in vitro. Eur J Med Res 17: 508-510, 1998.

11. Lahlou S, Figueierdo AF, Magalhaes PJ, et al: Cardiovascular effects of 1,8-cineole, a terpenoid oxide present in many plant essential oils, in normotensive rats. Can J Physiol Pharmacol 80: 1125-1131, 2002.

12. Moteki H, Hibasami H, Yamada Y, et al: Specific induction of apoptosis by 1,8-cineole in two human leukemia cell lines, but not $\mathrm{a}$ in human stomach cancer cell line. Oncol Rep 9: 757-760, 2002. 
13. Korzeniewski C and Callewaert DM: An enzyme-release assay for natural cytotoxicity. J Immunol Methods 64: 313-320, 1983

14. De Sousa AC, Alviano DS, Blank AF, et al: Melissa officinalis L. Essential oil: antitumoral and antioxidant activities. J Pharm Pharmacol 56: 677-681, 2004.

15. Silva J, Abebe W, Sousa SM, et al: Analgestic and anti-inflammatory effects of essential oils of Eucalyptus. J Ethnopharmacol 89: 277-283, 2003.

16. Williams LR, Stockley JK, Yan W, et al: Essential oils with high antimicrobial activity for therapeutic use. Int J Aromatherapy 8: 30-40, 1998.

17. Low D, Rawal BD and Griffin WJ: Antibacterial action of the essential oils of some Australian Myrtaceae with special references to the activity of chromatographic fractions of oil of Eucalyptus citriodora. Planta Med 26: 184-185, 1974.

18. Ramezani H, Singh HP, Batish DR, et al: Antifungal activity of the volatile oil of Eucalyptus citriodora. Fitoterapia 73 261-262, 2002.

19. Ashour HM: Antibacterial, antifungal and anticancer activities of volatile oils and extracts from stems, leaves and flowers of Eucalyptus sideroxylon and Eucalyptus torquata. Cancer Biol Ther 7: 399-403, 2008.

20. Altieri DC: Validating survivin as a cancer therapeutic target. Nat Rev Cancer 3: 46-54, 2003.

21. Carrasco RA, Stamm NB, Marcusson E, et al: Antisense inhibition of survivin expression as a cancer therapeutic. Mol Cancer Ther 10: 221-232, 2011

22. Rouse J, Cohen P, Trigon S, et al: A novel kinase cascade triggered by stress and heat shock that stimulates MAPKAP kinase-2 and phosphorylation of the small heat shock proteins. Cell 78: 1027-1037, 1994.
23. Han J, Lee JD, Bibbs L, et al: A MAP kinase targeted by endotoxin and hyperosmolarity in mammalian cells. Science 265: 808-811, 1994.

24. Lee JC, Laydon JT, McDonnell PC, et al: A protein kinase involved in the regulation of inflammatory cytokine biosynthesis. Nature 372: 739-746, 1994.

25. Freshney NW, Rawlinson L, Guesdon F, et al: Interleukin-1 activates a novel protein kinase cascade that results in the phosphorylation of Hsp27. Cell 78: 1039-1049, 1994.

26. Raingeaud J, Gupta S, Rogers JS, et al: Pro-inflammatory cytokines and environmental stress cause p38 mitogen-activated protein kinase activation by dual phosphorylation on tyrosine and threonine. J Biol Chem 270: 7420-7426, 1995.

27. Hsiao PW, Chang CC, Liu HF, et al: Activation of p38 mitogenactivated protein kinase by celecoxib oppositely regulates survivin and gamma-H2AX in human colorectal cancer cells. Toxicol Appl Pharmacol 222: 97-104, 2007.

28. Hsu YF, Sheu JR, Lin CH, et al: Trichostatin A and sirtinol suppressed survivin expression through AMPK and p38 MAPK in HT29 colon cancer cells. Biochem Biophys Acta 1820: 104-115, 2012.

29. Toker A and Yoeli-Lerner M: Akt signaling and cancer: surviving but not moving on. Cancer Res 66: 3963-3966, 2006.

30. Altomare DA and Testa JR: Perturbations of the AKT signaling pathway in human cancer. Oncogene 24: 7455-7464, 2005. 